\title{
Thermal properties of fluorinated graphene
}

\author{
Sandeep Kumar Singh, ${ }^{1}$ S. Goverapet Srinivasan, ${ }^{2}$ M. Neek-Amal, ${ }^{1,3,}{ }^{*}$ S. Costamagna,,${ }^{1,4}$ Adri C. T. van Duin, ${ }^{2}$ and \\ F. M. Peeters ${ }^{1}$ \\ ${ }^{1}$ Departement Fysica, Universiteit Antwerpen, Groenenborgerlaan 171, B-2020 Antwerpen, Belgium \\ ${ }^{2}$ Department of Mechanical and Nuclear Engineering, Pennsylvania State University, University Park, Pennsylvania 16801, USA \\ ${ }^{3}$ Department of Physics, Shahid Rajaee Teacher Training University, Lavizan, Tehran 16785-136, Iran \\ ${ }^{4}$ Facultad de Ciencias Exactas Ingeniería y Agrimensura, Universidad Nacional de Rosario and Instituto de Física Rosario, \\ Bv. 27 de Febrero 210 bis, 2000 Rosario, Argentina
}

(Received 8 January 2013; revised manuscript received 25 February 2013; published 29 March 2013)

\begin{abstract}
Large-scale atomistic simulations using the reactive force field approach are implemented to investigate the thermomechanical properties of fluorinated graphene (FG). A set of parameters for the reactive force field potential optimized to reproduce key quantum mechanical properties of relevant carbon-fluorine cluster systems are presented. Molecular dynamics simulations are used to investigate the thermal rippling behavior of FG and its mechanical properties and compare them with graphene, graphane and a sheet of boron nitride. The mean square value of the height fluctuations $\left\langle h^{2}\right\rangle$ and the height-height correlation function $H(q)$ for different system sizes and temperatures show that FG is an unrippled system in contrast to the thermal rippling behavior of graphene. The effective Young's modulus of a flake of fluorinated graphene is obtained to be $273 \mathrm{~N} / \mathrm{m}$ and $250 \mathrm{~N} / \mathrm{m}$ for a flake of FG under uniaxial strain along armchair and zigzag directions, respectively.
\end{abstract}

PACS number(s): 72.80.Vp, 68.65.Pq, 73.22.Pr

\section{INTRODUCTION}

The fascinating properties of single-layer graphene (GE) have triggered a broad interest in the solid state physics community. ${ }^{1-5}$ Despite its high electron mobility, ${ }^{6}$ the zero band gap defies its employment in nano transistors where it is desirable to have a large on-off ratio between conducting and nonconducting states. A band gap can be induced by the addition of adatoms, which changes locally the hybridization of the carbon (C) atoms, but also modifies the electron mean free path affecting the electron transport properties. Hydrogen $(\mathrm{H})$ and fluorine $(\mathrm{F})$ are two well-tested candidates, ${ }^{7-10}$ which lead to a large band gap opening. Graphane (GA, hydrogenated graphene) and fluorographene (FG) have been studied both experimentally and theoretically ${ }^{11-14}$ to engineer the band gap.

When $\mathrm{H}$ or $\mathrm{F}$ atoms are attached to the $\mathrm{C}$ atoms of GE, the bonds transit from an $s p^{2}$ to an $s p^{3}$ hybridization, which turns the conjugated graphitic C-C bonds into single $\mathrm{C}-\mathrm{C}$ bonds. In the fully covered cases both GA and FG are insulating materials 7,8 and the structure changes locally the planar shape of GE into an angstrom scale out-of-plane buckled shaped membrane ${ }^{15}$ known as chair configuration. ${ }^{16,17}$

From its potential applications in nanotechnology, FG is a more favorable material than GA since the C-F bonds are energetically more stable than the C-H bonds. ${ }^{13,15,17,18}$ Fluorographene has a very large temperature-dependent resistance and when the fluorine content is increased it induces large changes in the electron transport. ${ }^{19}$ As in GE, it is expected that temperature also affects strongly the lattice structure and the mechanical properties of FG.

According to the Mermin-Wagner theorem, ${ }^{20}$ thermal excited ripples in two-dimensional-like materials (GE, bilayer GE, GA, and FG) have to play an important role in the stability of the membrane. While in GE and bilayer GE the corrugations are well described within the theory of two-dimensional continuous membranes, ${ }^{21,22}$ for GA instead, we recently found that the angstrom scale buckling of the carbon layer of GA prevents the formation of intrinsic long wavelength thermal ripples for temperatures up to at least $900 \mathrm{~K} .{ }^{23}$

Since the $\mathrm{C}$ atom has a higher (lower) electronegativity than $\mathrm{H}(\mathrm{F})$, it will take (give) away charge from the $\mathrm{H}(\mathrm{F})$ atom and consequently transforms the resulting $\mathrm{C}-\mathrm{H}$ and $\mathrm{C}-\mathrm{F}$ covalent bonds into $s p^{3}$ bonds. Therefore, it is expected that similar rippling effects as in GA will occur in FG although the C-F bonds are somewhat stronger than the $\mathrm{C}-\mathrm{H}$ bonds. The latter is due to the larger amount of charge that is shifted from $\mathrm{C}$ to $\mathrm{F}$ as compared to the one from $\mathrm{H}$ to $\mathrm{C} .{ }^{13}$ However in order to simulate large-scale FG samples an appropriate force field is needed, which describes the true chemical bond in C-F. Indeed, the absence of such a suitable interatomic potential for C-F restricted most of the recent studies to $a b$ initio calculations of their electronic properties using a small computational unit cell.

Reactive force field (ReaxFF) potential serves to describe both bond and nonbond interactions in solids. Recently, such potentials were parameterized and were well tested for different kinds of structures, e.g., hydrocarbons, ${ }^{24}$ carbon allotropes ${ }^{25}$ etc. In this study we present a set of parameters for ReaxFF, appropriate for structures with C-F bonds. Using molecular dynamics (MD) simulations over large-scale samples we study the thermal corrugations of FG and compare the results with those found for GA, GE, and BN. We show that fully covered FG follows the same trend as GA and does not develop long-wavelength ripples or significant corrugation. The bending rigidity $\kappa$ of $\mathrm{FG}$ is found to be larger than the one of GE, GA, and BN. Furthermore, $\kappa$ turns out to be temperature independent. Our results indicate that long-wavelength ripples are instead present in partial covered FG samples with a larger amplitude as compared to GA.

The paper is organized as follows. In Sec. II, we introduce a new set of parameters for the ReaxFF potential of the C-F covalent bond. Then, in Sec. III using the introduced parameters, we analyze the thermal rippling behavior. Here, 
we consider both fully and partially covered graphene sheets by $\mathrm{F}$ atoms. All the results are compared with those previously found for graphane. We also estimate the effective Young's modulus of FG flakes. We conclude the paper in Sec. IV.

\section{REAXFF POTENTIAL FOR FLUOROGRAPHENE}

$\mathrm{ReaxFF}^{24}$ is a general bond-order-dependent potential that uses a relationship between bond distance and bond order on the one hand and a relationship between bond order and bond energy on the other hand to describe bond formation and dissociation. Many-body interactions such as the valence angle and torsional interactions are formulated as a function of bond order so that their energy contributions vanish smoothly upon bond dissociation. Nonbonded interactions, namely Coulomb and van der Waals interactions, are calculated between every pair of atoms irrespective of their connectivity. Excessively close-range interactions are avoided by shielding. ReaxFF uses the geometry-dependent charge calculation scheme (EEM scheme) of Mortier et al. ${ }^{26}$ The system energy in ReaxFF consists of a sum of terms

$$
\begin{aligned}
E_{\mathrm{sys}}= & E_{\mathrm{bond}}+E_{\text {under }}+E_{\mathrm{over}}+E_{\mathrm{lp}}+E_{\mathrm{val}}+E_{\mathrm{pen}} \\
& +E_{\text {tors }}+E_{\text {conj }}+E_{\mathrm{vdWaals}}+E_{\text {Coulomb }} .
\end{aligned}
$$

A detailed description of each of these terms and their functional forms can be found in the original work. ${ }^{24}$ The reactive force field for $\mathrm{C} / \mathrm{F}$ containing systems was developed by parameterizing the potential against DFT data obtained at the B3LYP/6-31g** level of theory (which is implemented in Schrödinger, ${ }^{27}$ which is an electronic structure package) for various quantities such as fluorine and carbon atom charges in $\mathrm{H}_{3} \mathrm{C}-\mathrm{CF}_{2}-\mathrm{CH}_{3}, \mathrm{C}-\mathrm{F}$ and $\mathrm{C}-\mathrm{C}$ bond lengths in $\mathrm{H}_{3} \mathrm{C}$ $\mathrm{CF}_{2}-\mathrm{CH}_{3}$, and $\mathrm{H}_{3} \mathrm{C}-\mathrm{CF}\left(\mathrm{CH}_{3}\right)-\mathrm{CH}_{3}, \mathrm{~F}-\mathrm{F}$ bond length in the $\mathrm{F}_{2}$ molecule, potential energy curve for $\mathrm{C}-\mathrm{F}$ bond dissociation in $\mathrm{H}_{3} \mathrm{C}-\mathrm{CF}_{2}-\mathrm{CH}_{3}, \mathrm{~F}-\mathrm{C}-\mathrm{F}$ angle bending in $\mathrm{H}_{3} \mathrm{C}-\mathrm{CF}_{2}-\mathrm{CH}_{3}$, $\mathrm{C}-\mathrm{C}-\mathrm{F}$ angle bending in $\mathrm{H}_{3} \mathrm{C}-\mathrm{CF}_{2}-\mathrm{CH}_{3}$ and $\mathrm{F}-\mathrm{C}-\mathrm{C}-\mathrm{F}$ dihedral twisting in $\mathrm{F}_{2} \mathrm{C}=\mathrm{CF}_{2}$, along with various chemical reactions involving fluoroalkanes and fluoroalkenes. The results of
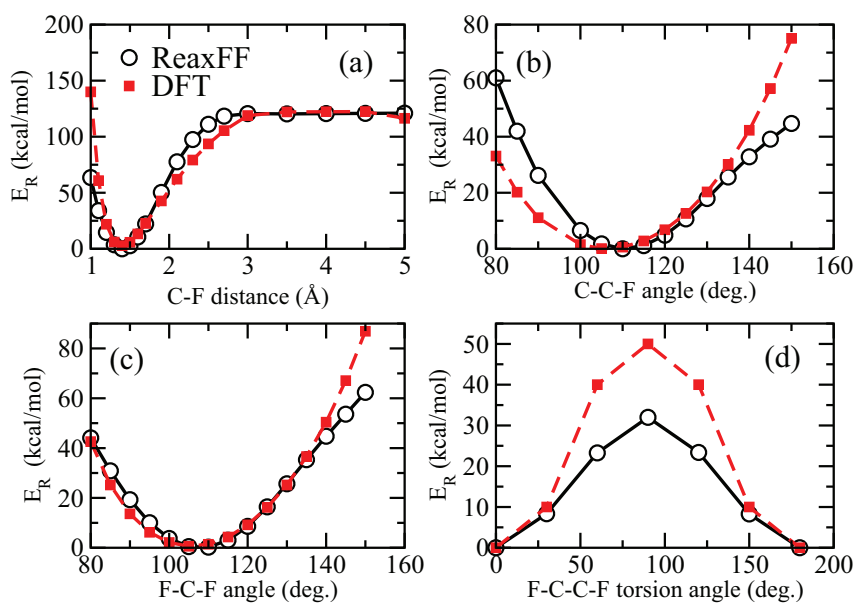

FIG. 1. (Color online) Comparison between DFT (solid squares) and ReaxFF (open circles) results for: (a) $\mathrm{C}-\mathrm{F}$ bond dissociation in $\mathrm{H}_{3} \mathrm{C}-\mathrm{CF}_{2}-\mathrm{CH}_{3}$ (b) $\mathrm{C}-\mathrm{C}-\mathrm{F}$ angle bending in $\mathrm{H}_{3} \mathrm{C}-\mathrm{CF}\left(\mathrm{CH}_{3}\right)-\mathrm{CH}_{3}$, (c) F-C-F angle bending in $\mathrm{H}_{3} \mathrm{C}-\mathrm{CF}_{2}-\mathrm{CH}_{3}$, and (d) F-C-C-F dihedral twisting in $\mathrm{F}_{2} \mathrm{C}=\mathrm{CF}_{2}$.
TABLE I. Comparison of equilibrium geometrical parameters between ReaxFF and DFT.

\begin{tabular}{lcr}
\hline \hline & DFT & ReaxFF \\
\hline $\mathrm{F}_{2}$ bond length & $1.43 \AA$ & $1.4012 \AA$ \\
C-F bond length in $\mathrm{H}_{3} \mathrm{C}-\mathrm{CF}_{2}-\mathrm{CH}_{3}$ & $1.3841 \AA$ & $1.4057 \AA$ \\
C-F bond length in $\mathrm{H}_{3} \mathrm{C}-\mathrm{CF}\left(\mathrm{CH}_{3}\right)-\mathrm{CH}_{3}$ & $1.3841 \AA$ & $1.4158 \AA$ \\
Nonbonding C-F distance in $\mathrm{CF}_{2}$ dimer & $2.00 \AA$ & $2.4471 \AA$ \\
F-C-F angle in $\mathrm{H}_{3} \mathrm{C}-\mathrm{CF}_{2}-\mathrm{CH}_{3}$ & $105.65^{\circ}$ & $107.2197^{\circ}$ \\
C-C-F angle in $\mathrm{H}_{3} \mathrm{C}-\mathrm{CF}\left(\mathrm{CH}_{3}\right)-\mathrm{CH}_{3}$ & $106.2^{\circ}$ & $109.9625^{\circ}$ \\
\hline \hline
\end{tabular}

the force field training are presented in Figs. 1(a)-1(d) and in Table I. Figure 2 depicts the geometrical quantities relevant to Figs. 1(a)-1(d). It can be seen from Table I that ReaxFF reproduces closely the DFT-based equilibrium geometries for various compounds. ReaxFF predicts $F_{2}$ dissociation energy of $36.6 \mathrm{kcal} / \mathrm{mol}$, in very good agreement with the DFT value of $37 \mathrm{kcal} / \mathrm{mol}$. Figure 1(a) shows that the ReaxFF-based potential energy curve for the $\mathrm{C}-\mathrm{F}$ bond dissociation in $\mathrm{H}_{3} \mathrm{C}-\mathrm{CF}_{2}-\mathrm{CH}_{3}$ closely follows the DFT-based potential energy curve. ReaxFF is able to predict very precisely the equilibrium C-F bond length (see Table I) and the C-F bond dissociation energy. Similarly the force field can closely reproduce the DFT-based potential energy curve and the equilibrium geometry (see Table I) for $\mathrm{C}-\mathrm{C}-\mathrm{F}$ angle bending and the C-F-C angle bending as shown in Figs. 1(b)-1(c). Figure 1(d) shows the variation of the potential energy upon F-C-C-F dihedral angle twisting. Though ReaxFF predicts the correct trend, the torsional rotation barrier in ReaxFF is around $18 \mathrm{kcal} / \mathrm{mol}$ lower than that predicted by DFT. Overall, the ReaxFF force field for $\mathrm{C} / \mathrm{F}$ systems can closely reproduce the DFT-based energies and geometries for a number of molecules and reactions. This force field will now be employed in large-scale fully reactive molecular dynamics simulation of $\mathrm{C} / \mathrm{F}$ containing systems.

In the next section we study the thermal structural fluctuations and mechanical properties of a single layer of FG using large-scale atomistic simulations employing the presented (a)

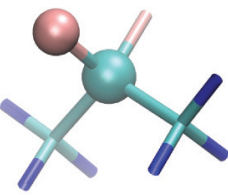

(c)

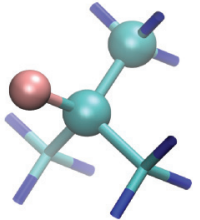

(b)

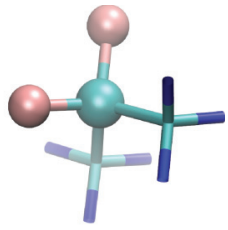

(d)

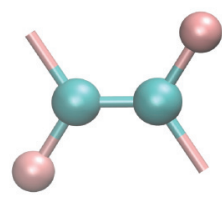

FIG. 2. (Color online) The molecules used for parameterizing the ReaxFF force field in this study. (a) $\mathrm{C}-\mathrm{F}$ bond in $\mathrm{H}_{3} \mathrm{C}-\mathrm{CF}_{2}-\mathrm{CH}_{3}$, (b) F-C-F angle in $\mathrm{H}_{3} \mathrm{C}-\mathrm{CF}_{2}-\mathrm{CH}_{3}$, (c) $\mathrm{C}-\mathrm{C}-\mathrm{F}$ angle in $\mathrm{H}_{3} \mathrm{C}-\mathrm{CF}\left(\mathrm{CH}_{3}\right)$ $\mathrm{CH}_{3}$, and (d) F-C-C-F dihedral angle in $\mathrm{F}_{2} \mathrm{C}=\mathrm{CF}_{2}$. The atoms constituting the geometric parameters are represented by balls while the rest of the atoms are represented by sticks. F atoms are colored brown, $\mathrm{C}$ atoms are colored green and $\mathrm{H}$ atoms are colored blue. 
ReaxFF parameters. These parameters were implemented in the large-scale atomic/molecular massively parallel simulator package LAMMPS. ${ }^{28,29}$

\section{RESULTS}

\section{A. Thermal rippling behavior of FG}

In order to study the rippling behavior of FG we considered a square shaped computational unit cell of FG with both armchair and zigzag edges in the $x$ and $y$ directions. Partial fluorine contents of $10 \%, 50 \%, 70 \%, 90 \%$, and the fully covered $100 \%$ case (with a total number of $N=17280$ atoms) were studied. In our simulation we employed the NPT ensemble with $P=0$ using the Nosé-Hoover thermostat and varied the temperature from $10 \mathrm{~K}$ to $900 \mathrm{~K}$. Figure 3 shows the obtained buckled shape of fully fluorinated sample after relaxation, which is in agreement with recent DFT results. ${ }^{13}$

One would expect that the thermal excited ripples in FG can be described by membrane theory for a two-dimensional (2D) continuous membrane. ${ }^{30}$ This theory, described in a series of related works, ${ }^{23,31-33}$ is supposed to be universal and independent of the atomic scale details of the membrane. The main predictions of this theory are as follows. Let $h$ be the out-of-plane displacement of a given atom of a sheet, then the Fourier transform of the height-height correlation function is in the harmonic approximation given by

$$
H(q)=\left\langle|h(q)|^{2}\right\rangle=\frac{N k_{B} T}{\kappa S_{0} q^{4}},
$$

where $q$ is the wave vector, $N$ is the number of atoms, $S_{0}$ is the surface area per atom, $\kappa$ is the bending rigidity of the membrane, and $k_{B}$ is the Boltzmann constant.

In the large-wavelength limit, anharmonic couplings between bending and stretching modes are important resulting in a renormalization of the $q$-dependent behavior

$$
H(q)=\frac{N k_{B} T}{\kappa S_{0} q^{4-\eta}} .
$$

where $\eta$ is an universal scaling exponent, which is about $\approx$ $0.85 .^{34-36}$

In order to compare our results for FG with other twodimensional materials, we used a modified Tersoff potential (which is an ordinary defined potential in the LAMMPS package ${ }^{29}$ ) according to the parameters proposed by Sevik et al. for the h-BN sheet. ${ }^{37}$ To simulate GE and GA we have used the AIREBO potential, ${ }^{38}$ which is suitable for simulating hydrocarbons.

Recently, we found that in GA, $H(q)$ acquires a strong renormalization for small wave vectors $q$ and the layer remains

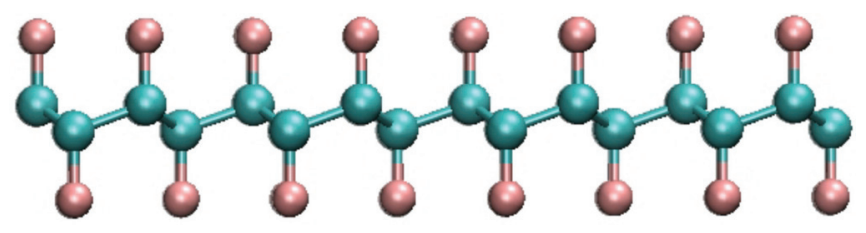

FIG. 3. (Color online) (a) Side view of the buckled structure, known as chair configuration, of fully fluorinated graphene. The averaged bond angle, and $\mathrm{C}-\mathrm{C}$ and $\mathrm{C}-\mathrm{F}$ distances, are respectively $109.5^{\circ}, d_{\mathrm{C}-\mathrm{C}}=1.58 \AA$ and $d_{\mathrm{C}-\mathrm{F}}=1.41 \AA$ at room temperature. almost flat even for temperatures as high as $900 \mathrm{~K} .{ }^{23}$ Here we will analyze the thermal rippling behavior of FG and compare it with GA. A comparison with GE and $\mathrm{BN}$ single layers, which behave as 2D membranes ${ }^{31,39}$ will also be presented. $H(q)$ for FG was calculated following the steps described in our previous work. ${ }^{23}$

Starting from a pure GE sheet, the variation of the heightheight correlation function $H(q)$ at room temperature for different partial fluorine contents is shown in Fig. 4(a). The curves were shifted for a better comparison. We found that while for 10 to $90 \%$ coverage, $H(q)$ follows Eq. (2) up to small $q$ values, which is similar to the case of GE. ${ }^{32}$ But, for fully FG at $q^{*} \approx 0.2 \AA^{-1}, H(q)$ deviates from the harmonic law (solid line) and approaches roughly a constant value similar to what was previously found for GA. ${ }^{23}$ In the inset of Fig. 4(a) we show the square average of the out-of-plane fluctuations $\left\langle h^{2}\right\rangle$ at $300 \mathrm{~K}$. Notice that the out-of-plane fluctuations for partially
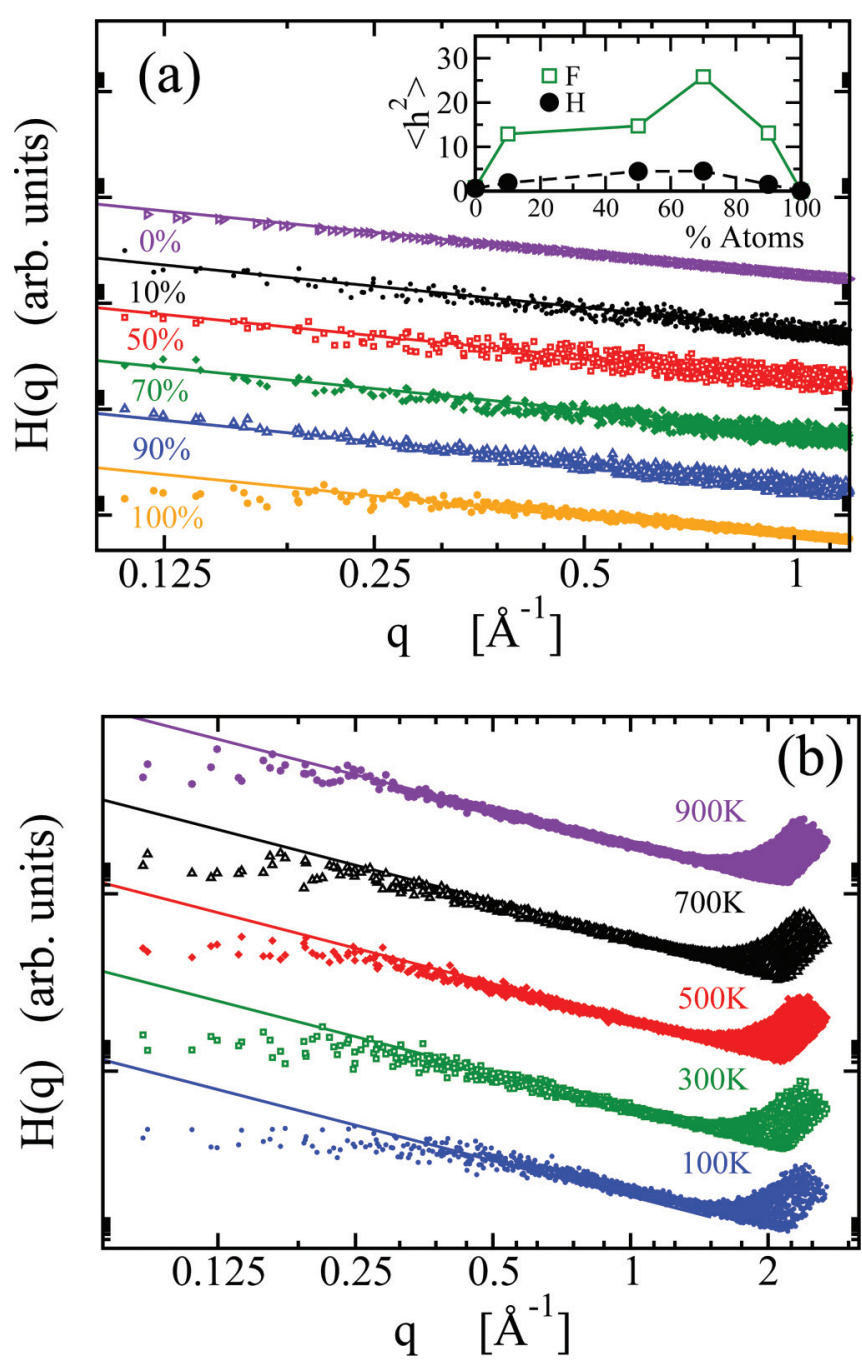

FIG. 4. (Color online) (a) Log-log plot of $H(q)$ for different coverage of $\mathrm{F}$ atoms at $T=300 \mathrm{~K}$. The solid lines show the harmonic $q^{-4}$ behavior valid in the limit of large $q$ values. Note the strong deviation, starting roughly at $q^{*} \approx 0.2 \AA^{-1}$ in the limit of long wavelengths, for the case of fully fluorinated graphene. The variation of $\left\langle h^{2}\right\rangle$ is shown in the inset. (b) $H(q)$ for fully fluorinated FG at different temperatures. 


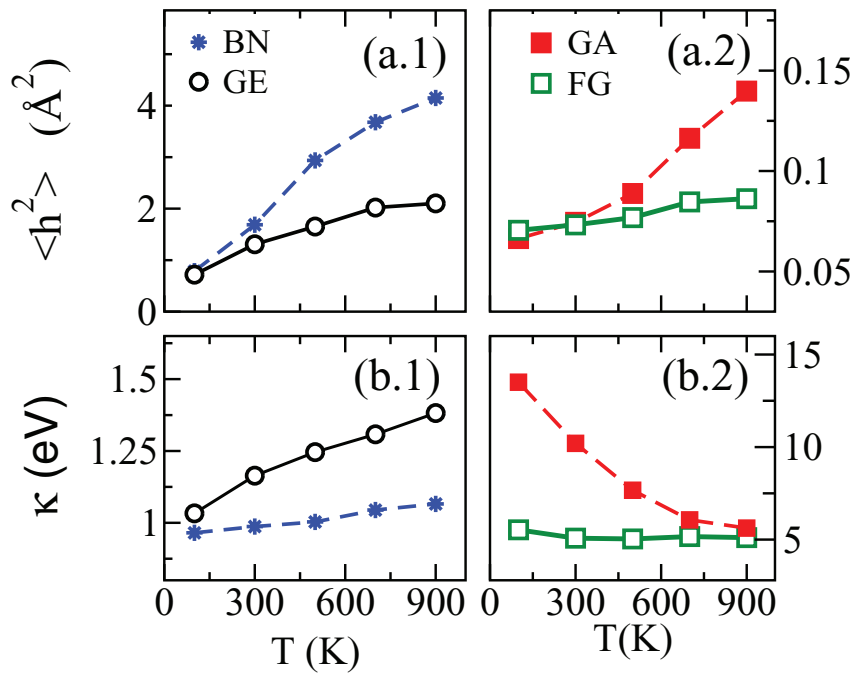

FIG. 5. (Color online) Variation of (a) $\left\langle h^{2}\right\rangle$, and (b) $\kappa$ against temperature for FG (open squares), GA (filled squared), GE (open circles), and $\mathrm{BN}$ (stars).

covered samples are considerably larger for FG than for GA. The temperature dependence of $H(q)$ for fully fluorinated graphene is shown in Fig. 4(b). Irrespective of temperature, the short wavelength limit of $H(q)$ tends always approximately to a constant value. The characteristic $q$ value where $H(q)$ deviates from the harmonic approximation result decreases with increasing temperature.

The renormalization of $H(q)$ for long wavelengths indicates the suppression of large out-of-plane height fluctuations. In Fig. 5(a) we compare the behavior of $\left\langle h^{2}\right\rangle$ against temperature for GE, BN [Fig. 5(a.1)], FG and GA [Fig. 5(a.2)]. Notice that $\left\langle h^{2}\right\rangle$ increases from $0.7 \AA^{2}$ up to $4 \AA^{2}$ in BN and from $0.7 \AA^{2}$ up to $2 \AA^{2}$ in GE when temperature is varied from $10 \mathrm{~K}$ up to $900 \mathrm{~K}$. Due to the absence of long wavelength ripples, $\left\langle h^{2}\right\rangle$ remains approximately constant for GA and FG, and the variations are smaller than those for $\mathrm{BN}$ and GE, over the same temperature range. The temperature dependence of the bending rigidity $\kappa$, computed from the harmonic part of $H(q)$ is shown in Fig. 4(b). Note that the larger magnitude for GA and $\mathrm{FG}$ is a consequence of the smaller corrugations present in these materials. We also find the opposite temperature dependence for BN and GE when compared with GA and FG. In this sense GE and BN behave anomalously. The corresponding bending rigidity and $\left\langle h^{2}\right\rangle$ at room temperature for GE, GA, and FG are listed in Table II.

Density functional calculations for band structure of FG (and GA) $)^{40}$ show that the acoustic out-of-plane modes (ZA)

TABLE II. Comparison of AIREBO and ReaxFF for the bending rigidity and $\left\langle h^{2}\right\rangle$ for GE, GA and FG at $300 \mathrm{~K}$.

\begin{tabular}{lccccc}
\hline \hline & \multicolumn{2}{c}{ AIREBO } & & \multicolumn{2}{c}{ ReaxFF } \\
\cline { 2 - 3 } \cline { 5 - 6 } & $\kappa(e V)$ & $\left\langle h^{2}\right\rangle\left(\AA^{2}\right)$ & & $\kappa(e V)$ & $\left\langle h^{2}\right\rangle\left(\AA^{2}\right)$ \\
\hline GE & 1.165 & 1.307 & 1.16 & 0.627 \\
GA & 10.19 & 0.070 & & 7.26 & 0.074 \\
GF & - & - & 5.07 & 0.073 \\
\hline \hline
\end{tabular}

in FG (and GA) are different from that of GE. The most important difference from GE is the decoupled optical and acoustic bands in FG and GA close to the $\Gamma$ point. The light $\mathrm{H}$ atom contributes to the highest phonon frequencies, which is not the case for $\mathrm{F}$ atoms. It is also seen that the $\mathrm{ZA}$ modes close to the $\Gamma$ point for FG (and GA) are not well fitted by a quadratic function in contrast to the GE case. This is clear indication of anharmonicity. In summary, the atomistic details of the structure of FG is more complicated and therefore more details of this structure should be included in any continuum theory.

Before ending this section, note that as we discussed in our previous work, ${ }^{23}$ the scaling with the system size present in GE is no longer valid for FG and GA $\left(\left\langle h^{2}\right\rangle\right.$ in FG and GA is almost constant irrespective to the system size). The lower wavelengths $(q)$ adopted for the calculation of $H(q)$ are equal to $q_{\mathrm{x}-\min }=\frac{2 \pi}{l_{x}}$ and $q_{\mathrm{y}-\min }=\frac{2 \pi}{l_{y}}$ and represent the computational cutoffs of possible large wavelength ripples where $l_{x}$ and $l_{y}$ are the dimension of the system. Notice that deviation from the harmonic behavior takes place at larger value of $q$ and hence this effect can not be a finite size effect and is instead an intrinsic phenomenon of the material.

\section{B. Effective Young's modulus}

In order to study the mechanical stiffness we consider an FG flake with dimension $l_{x} \times l_{y}=15 \times 15 \mathrm{~nm}^{2}$. Before the stretching process, the sample is equilibrated for 5 ps (i.e., 50000 time steps). Stretching direction is always along $x$ and the uniaxial strain is applied within the NPT ensemble ${ }^{41}$ where the pressure is slowly increased, i.e., $2 \mathrm{GPas} / \mathrm{ps}$. In this section the lateral edges (in the $y$ direction) were taken as the armchair direction having both free (FBC) and periodic boundary conditions (PBC). We kept temperature fixed at $T=10 \mathrm{~K}$.

The total strain energy per atom of the strained flake can be written as a function of the imposed strain $(\epsilon)$

$$
E_{T}\left(l_{y}, \epsilon\right)=E_{0}+\frac{S_{0}}{l_{y}} \gamma(\epsilon)+\frac{S_{0}}{2} Y \epsilon^{2},
$$

where $E_{0}$ is the energy of the infinite planar undeformed flake, $\gamma(\epsilon)$ is the excess edge energy, and $Y$ is Young's modulus $(Y)$ of the flake.

For nanoribbons with no lateral edges we have $\gamma=0$ (assuming that the longitudinal edges that are fixed make no contribution). This is due to the fact that free edges increase the energy due to buckling and bond-order changes. ${ }^{42}$ Recently Lu et al. used the Brenner potential ${ }^{43}$ in molecular dynamics simulations and studied the excess edge energy of graphene nanoribbons as a function of width and chirality. ${ }^{42}$ Our systems are different from those of Ref. 42. In contrast to Ref. 42 we are not interested in effects due to the edge energy effect and the size dependence. We rewrite Eq. (3) in the following as an effective Young's modulus, which qualitatively gives a good description of the mechanical stiffness of all the examined 2D materials. Nevertheless our results are in qualitative agreement with those reported by Lu at al, i.e., increasing of total energy for the FBC case as compared to a nanoribbon. Assuming a quadratic relation for $\gamma(\epsilon)=\frac{l_{y}}{2} \epsilon^{2}$ valid for small $\epsilon$, the simplest method to estimate Young's modulus is by fitting 


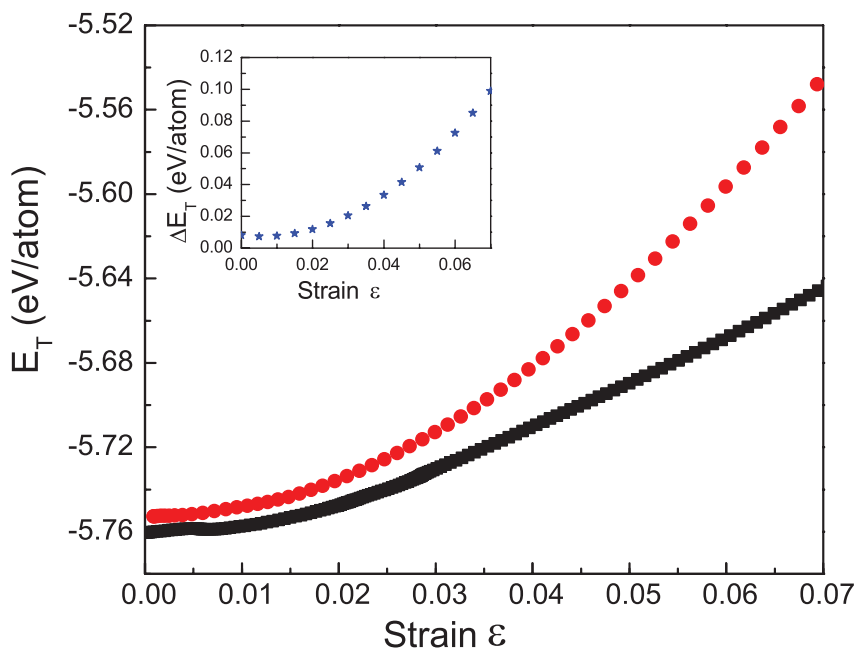

FIG. 6. (Color online) (a) Variation of total energy against uniaxial strain for FG subjected to free boundary condition (FBC) and periodic boundary condition (PBC) for the lateral edges, i.e., the dashed and solid curves respectively. The inset shows the difference between the two energy curves.

the quadratic function to the total energy (per area)

$$
E_{T}=E_{0}+\frac{1}{2} Y_{\text {eff }} \epsilon^{2},
$$

where $Y_{\text {eff }}$ is the effective Young's modulus of the system. Using aforementioned fitting process we found $Y_{\text {eff }}$ respectively for a flake with armchair and zigzag FG, to be $273 \mathrm{~N} / \mathrm{m}$ and $250 \mathrm{~N} / \mathrm{m}$. Notice that the experimental result is $100 \mathrm{~N} / \mathrm{m}$ for not perfect $\mathrm{FG}^{8}$ while the DFT result is $250 \mathrm{~N} / \mathrm{m} .{ }^{18}$ The latter disagreement between theory and experiment may be explained due to the fact that in experimental samples the fluorine-to-carbon ratio is larger than unity, i.e., $1.1,{ }^{8}$ because of the presence of defects. Such defects become active regions which can adsorb the free $\mathrm{F}$ (and even $\mathrm{H}$ ) atoms. Therefore, in the defected parts more $\mathrm{F}$ atoms will be found, which is responsible for a $\mathrm{F} / \mathrm{C}$ ratio larger than one.

In order to understand the effect of the different boundary conditions, we depict in Fig. 6 the variation of $E_{T}$ per atom with $\epsilon$ for flakes with both FBC (dashed lines) and PBC, i.e., nanoribbon (solid lines). It is seen that for flakes with FBC the free edges result in an increase of the energy. The inset shows the difference between two curves, i.e., $\Delta E_{T}=E_{\mathrm{FBC}}-E_{\mathrm{PBC}}$, which is positive. Because the free boundaries have many dangling bonds, which are not saturated by $\mathrm{F}$ atoms it results in extra energy. This can also occur in other systems, e.g., graphene. ${ }^{44}$ Notice that for the studied low temperature here, i.e., $T=10 \mathrm{~K}$ we do not expect that bond reconstruction at the edges is important. Notice that even by saturating all the bonds by $\mathrm{F}$, still the change in the bond order term in ReaxFF (due to different chemical environment of the boundary atoms) results in higher energy as compared to PBC.

Furthermore, both FBC and $\mathrm{PBC}$ results exhibit a quadratic behavior, which is an indication of the harmonic regime for the applied uniaxial stain. As is clear from the inset of Fig. 6, the difference between the two curves is increasing with applied strain. This is due to the deviation from equilibrium for the $\mathrm{C}-\mathrm{F}$ bonds, C-C-F (F-C-F) bond angles, and the dihedral angles (F-C-C-F torsion angle) of the free edge atoms. The larger the strain (and the larger the length of ribbon), the larger the deviation from equilibrium for the bonds and the angles. In the PBC case there is no such edge effect but, nevertheless, because of the fixing of the two longitudinal ends (the edges that are under uniaxial stress) the energy variation of the PBC system should be different from that of an infinite FG, which is periodic in both directions while it is under tension from the armchair direction. The fixed longitudinal ends do not have any effect in our results because both FBC and PBC have the same contributions.

\section{CONCLUSION}

We provided a set of parameters for the ReaxFF potential for the C-F covalent bond and tested it on various molecules. Subsequently, molecular dynamics simulations were used to investigate the thermal rippling behavior and the mechanical response of fluorographene (FG) under uniaxial stress. The obtained results are compared with those for graphene (GE), graphane (GA), and hexagonal boron nitride sheet $(\mathrm{BN})$. We found that fluorographene remains a flat sheet similar to graphane even at high temperature, i.e., up to $900 \mathrm{~K}$. The bending rigidity of FG is found to be independent of temperature and its Young's modulus is in good agreement with experiment.

\section{ACKNOWLEDGMENTS}

M.N.-A. is supported by the EU-Marie Curie IIF postdoc Fellowship/299855. This work is supported by the ESFEurographene project CONGRAN, the Flemish Science Foundation (FWO-Vl), and the Methusalem Foundation of the Flemish Government. S.G.S. and A.C.T.vD. acknowledge support by the Air Force Office of Scientific Research (AFOSR) under Grant No. FA9550-10-1-0563. *neekamal@srttu.edu

${ }^{1}$ K. S. Novoselov, A. K. Geim, S. V. Morozov, D. Jiang, Y. Zhang, S. V. Dubonos, I. V. Grigorieva, and A. A. Firsov, Science 306, 666 (2004).

${ }^{2}$ A. K. Geim and K. S. Novoselov, Nature Mater. 6, 183 (2007).

${ }^{3}$ C. Berger, Z. Song, T. Li, A. Y. Ogbazghi, R. Feng, Z. Dai, A. N. Marchenkov, E. H. Conrad, P. N. First, and W. A. de Heer, Science 312, 1191 (2006).

${ }^{4}$ M. I. Katsnelson, K. S. Novoselov, and A. K. Geim, Nature Phys. 2, 620 (2006).
${ }^{5}$ H. Şahin, R. T. Senger, and S. Ciraci, J. Appl. Phys. 108, 074301 (2010).

${ }^{6}$ A. H. Castro Neto, F. Guinea, N. M. R. Peres, K. S. Novoselov, and A. K. Geim, Rev. Mod. Phys. 81, 109 (2009).

${ }^{7}$ D. C. Elias, R. R. Nair, T. M. G. Mohiuddin, S. V. Morozov, P. Blake, M. P. Halsall, A. C. Ferrari, D. W. Boukhvalov, M. I. Katsnelson, A. K. Geim, and K. S. Novoselov, Science 323, 610 (2009).

${ }^{8}$ R. R. Nair, W. Ren, R. Jalil, I. Riaz, V. G. Kravets, L. Britnell, P. Blake, F. Schedin, A. S. Mayorov, S. Yuan, M. I. Katsnelson, 
H. M. Cheng, W. Strupinski, L. G. Bulusheva, A. V. Okotrub, I. V. Grigorieva, A. N. Grigorenko, K. S. Novoselov, and A. K. Geim, Small 6, 2877 (2010).

${ }^{9}$ J. T. Robinson, J. S. Burgess, C. E. Junkermeier, S. C. Badescu, T. L. Reinecke, F. K. Perkins, M. K. Zalautdniov, J. W. Baldwin, J. C. Culbertson, P. E. Sheehan, and E. S. Snow, Nano Lett. 10, 3001 (2010).

${ }^{10}$ R. Zboril, F. Karlicky, A. B. Bourlinos, T. A. Steriotis, A. K. Stubos, V. Georgakilas, K. Safarova, D. Jancik, C. Trapalis, and M. Otyepka, Small 6, 2885 (2010).

${ }^{11}$ S. H. Cheng, K. Zou, F. Okino, H. R. Gutierrez, A. Gupta, N. Shen, P. C. Eklund, J. O. Sofo, and J. Zhu, Phys. Rev. B 81, 205435 (2010).

${ }^{12}$ F. Withers, M. Dubois, and A. K. Savchenko, Phys. Rev. B 82, 073403 (2010).

${ }^{13}$ O. Leenaerts, H. Peelaers, A. D. Hernández-Nieves, B. Partoens, and F. M. Peeters, Phys. Rev. B 82, 195436 (2010).

${ }^{14}$ D. K. Samarakoon, Z. Chen, C. Nicolas, and X. Q. Wang, Small 7, 965 (2011).

${ }^{15}$ D. W. Boukhvalov, M. I. Katsnelson, and A. I. Lichtenstein, Phys. Rev. B 77, 035427 (2008).

${ }^{16}$ M. H. F. Sluiter and Y. Kawazoe, Phys. Rev. B 68, 085410 (2003).

${ }^{17}$ J. O. Sofo, A. S. Chaudhari, and G. D. Barber, Phys. Rev. B 75, 153401 (2007).

${ }^{18}$ H. Şahin, M. Topsakal, and S. Ciraci, Phys. Rev. B 83, 115432 (2011).

${ }^{19}$ F. Withers, S. Russo, M. Dubois, and M. F. Craciun, Nanoscale Res. Lett. 6, 526 (2011).

${ }^{20}$ N. D. Mermin and H. Wagner, Phys. Rev. Lett. 17, 1133 (1966).

${ }^{21}$ K. V. Zakharchenko, J. H. Los, M. I. Katsnelson, and A. Fasolino, Phys. Rev. B 81, 235439 (2010).

${ }^{22}$ J. C. Meyer, A. K. Geim, M. I. Katsnelson, K. S. Novoselov, T. J. Booth, and S. Roth, Nature (London) 446, 60 (2007); D. A. Kirilenko, A. T. Dideykin, and G. Van Tendeloo, Phys. Rev. B 84, 235417 (2011).

${ }^{23}$ S. Costamagna, M. Neek-Amal, J. H. Los, and F. M. Peeters, Phys. Rev. B 86, 041408 (2012).
${ }^{24}$ A. C. T. van Duin, S. Dasgupta, F. Lorant, and W. A. Goddard, J. Phys. Chem. A 105, 9396 (2001).

${ }^{25}$ J. E. Mueller, A. C. T. van Duin, and W. A. Goddard III, J. Phys. Chem. C 114, 4939 (2010).

${ }^{26}$ W. J. Mortier, S. K. Ghosh, and S. Shankar, J. Am. Chem. Soc. 108, 4315 (1986).

${ }^{27}$ JAGUAR, version 7.8 (Schrödinger, New York, 2011).

${ }^{28} \mathrm{http}: / /$ lammps.sandia.gov

${ }^{29}$ S. J. Plimpton, J. Comput. Phys. 117, 1 (1995).

${ }^{30}$ D. Nelson, T. Piran, and S. Weinberg, Statistical Mechanics of Membrane and Surface (World Scientific, Singapore, 2004).

${ }^{31}$ A. Fasolino, J. H. Los, and M. I. Katsnelson, Nature Mater. 6, 858 (2007).

${ }^{32}$ S. Costamagna and A. Dobry, Phys. Rev. B 83, 233401 (2011).

${ }^{33}$ R. Roldán, A. Fasolino, K. V. Zakharchenko, and M. I. Katsnelson, Phys. Rev. B 83, 174104 (2011).

${ }^{34}$ P. Le Doussal and L. Radzihovsky, Phys. Rev. Lett. 69, 1209 (1992).

${ }^{35}$ J. H. Los, L. M. Ghiringhelli, E. J. Meijer, and A. Fasolino, Phys. Rev. B 72, 214102 (2005).

${ }^{36}$ J. H. Los, M. I. Katsnelson, O. V. Yazyev, K. V. Zakharchenko, and A. Fasolino, Phys. Rev. B 80, 121405 (2009).

${ }^{37}$ C. Sevik, A. Kinaci, J. B. Haskins, and T. Çağin, Phys. Rev. B 84, 085409 (2011)

${ }^{38}$ S. J. Stuart, A. B. Tutein, and J. A. Harrison, J. Chem. Phys. 112, 6472 (2000).

${ }^{39}$ S. K. Singh, M. Neek-Amal, S. Costamagna, and F. M. Peeters, Phys. Rev. B (to be published).

${ }^{40}$ H. Peelaers, A. D. Hernandez-Nieves, O. Leenaerts, B. Partoens, and F. M. Peeters, Appl. Phys. Lett. 98, 051914 (2011).

${ }^{41}$ Y. Zheng, N. Wei, Z. Fan, L. Xu, and Z. Huang, Nanotechnology 22, 405701 (2011).

${ }^{42}$ Q. Lu and R. Huang, Phys. Rev. B 81, 155410 (2010); J. Phys.: Condens. Matter 19, 054006 (2011).

${ }^{43}$ D. W. Brenner, O. A. Shenderova, J. A. Harrison, S. J. Stuart, B. Ni, and S. B. Sinnott, J. Phys.: Condens. Matter 14, 783 (2002).

${ }^{44}$ P. Koskinen, S. Malola, and H. Häkkinen, Phys. Rev. Lett. 101, 115502 (2008) 